\title{
Pharmacometric Models for Characterizing the Pharmacokinetics of Orally Inhaled Drugs
}

\author{
Jens Markus Borghardt, ${ }^{1}$ Benjamin Weber, ${ }^{2}$ Alexander Staab, ${ }^{2}$ and Charlotte Kloft ${ }^{1,3}$
}

Received 17 December 2014; accepted 25 March 2015; published online 7 April 2015

\begin{abstract}
During the last decades, the importance of modeling and simulation in clinical drug development, with the goal to qualitatively and quantitatively assess and understand mechanisms of pharmacokinetic processes, has strongly increased. However, this increase could not equally be observed for orally inhaled drugs. The objectives of this review are to understand the reasons for this gap and to demonstrate the opportunities that mathematical modeling of pharmacokinetics of orally inhaled drugs offers. To achieve these objectives, this review (i) discusses pulmonary physiological processes and their impact on the pharmacokinetics after drug inhalation, (ii) provides a comprehensive overview of published pharmacokinetic models, (iii) categorizes these models into physiologically based pharmacokinetic (PBPK) and (clinical data-derived) empirical models, (iv) explores both their (mechanistic) plausibility, and (v) addresses critical aspects of different pharmacometric approaches pertinent for drug inhalation. In summary, pulmonary deposition, dissolution, and absorption are highly complex processes and may represent the major challenge for modeling and simulation of PK after oral drug inhalation. Challenges in relating systemic pharmacokinetics with pulmonary efficacy may be another factor contributing to the limited number of existing pharmacokinetic models for orally inhaled drugs. Investigations comprising in vitro experiments, clinical studies, and more sophisticated mathematical approaches are considered to be necessary for elucidating these highly complex pulmonary processes. With this additional knowledge, the PBPK approach might gain additional attractiveness. Currently, (semi-)mechanistic modeling offers an alternative to generate and investigate hypotheses and to more mechanistically understand the pulmonary and systemic pharmacokinetics after oral drug inhalation including the impact of pulmonary diseases.
\end{abstract}

KEY WORDS: inhalation; mathematical models; physiologically based pharmacokinetic models; population pharmacokinetics; pulmonary absorption.

\section{INTRODUCTION}

The role of mathematical modeling to assess and understand pharmacokinetics (PK) in clinical drug development and therapeutic use has gained more recognition and importance over the last decades (1-3). However, the number of models characterizing the systemic and pulmonary PK of orally inhaled drugs is limited compared with other routes of administration, e.g., intravenous (IV) or oral. To understand the reasons for this gap and to demonstrate the opportunities that mathematical modeling of PK of inhaled drugs offers, this review provides a comprehensive overview of published PK models, categorizes the PK models into physiologically based PK (PBPK; PK parameters derived

\footnotetext{
${ }^{1}$ Department of Clinical Pharmacy and Biochemistry, Institute of Pharmacy, Freie Universitaet Berlin, Kelchstr. 31, 12169, Berlin, Germany.

${ }^{2}$ Department of Translational Medicine and Clinical Pharmacology, Boehringer Ingelheim Pharma GmbH \& Co. KG, Biberach, Germany.

${ }^{3}$ To whom correspondence should be addressed. (e-mail: charlotte.kloft@fu-berlin.de)
}

from physico-chemical characteristics of the inhaled drug and physiological characteristics) and empirical models (PK parameters estimated based on clinical data), and explores their (mechanistic) plausibility. To achieve these aims, firstly relevant physiological and anatomical characteristics of the lung as well as mechanistic aspects of oral drug inhalation were summarized. Secondly, a comprehensive literature review for modeling approaches describing the pulmonary and systemic PK after inhalation was performed; pulmonary PK refers to lung-specific kinetics before drug is being absorbed into the systemic circulation, such as pulmonary dissolution and pulmonary absorption, or mucociliary clearance, whereas systemic PK refers to the PK processes after drug is absorbed from the lung into the systemic circulation. Throughout this review, a clear distinction is made between PBPK models (often called bottom-up approach) and empirical PK models (often called top-down approach). Finally, the reviewed PK models were systematically evaluated in the context of physiological plausibility and critical issues pertinent to drug inhalation were discussed. It should be noted that this article and a recently published book chapter by Kandala and Hochhaus on "applied pharmacometrics in pulmonary diseases" (4) partially overlap in their scopes but 
overall can be considered complementary. The book chapter focuses on specific examples in pharmacokinetics and also expands to (systemic) pharmacodynamics (PD) after drug inhalation. This review article gives a comprehensive overview and categorization of published pharmacokinetic models after drug inhalation and does not include any pharmacodynamics.

\section{The Lung As Target for Drug Administration}

The lung is the organ that represents the barrier between air and blood. During oral inhalation, air is passing through the mouth, throat (oropharyngeal region), and subsequently the conducting airways until air is available for gas exchange in the alveolar space. The (conducting) airways can be differentiated into central and peripheral airways or characterized with specific airway generation numbers. Delivering drugs directly to the lung is utilized to either administer drugs to achieve efficacy by distribution to the target via systemic circulation or more frequently for local treatment of pulmonary diseases, such as asthma bronchiale or chronic obstructive pulmonary disease (COPD) $(5,6)$. When a drug is inhaled with the focus of local treatment, unbound pulmonary and systemic drug concentrations are associated with efficacy and systemic safety, respectively; thus, the goal is to maximize pulmonary and to minimize systemic (unbound) drug concentrations (7). To achieve the same pulmonary drug concentrations after IV or oral drug administration, higher oral and IV doses would typically be required compared with inhalation. Higher oral and IV doses, however, would result in higher plasma concentrations associated with a higher probability of systemic adverse drug reactions $(8,9)$, since drug deposited in the lung is typically not instantaneously absorbed to systemic circulation. Hence, for drugs without instantaneous pulmonary absorption, oral drug inhalation typically results in a favorable benefit/(systemic) risk ratio (10).

In contrast, when the purpose of drug inhalation is systemic treatment, plasma drug concentrations are associated with both efficacy and safety. Nonetheless, pulmonary drug concentrations might be important to evaluate pulmonary adverse drug reactions, e.g., for inhaled insulin (11).

\section{Inhalation and Pulmonary Pharmacokinetic Processes}

To understand both the pulmonary and the systemic PK after drug inhalation, a thorough understanding of the different (PK) processes in the lung is crucial. When a drug is inhaled, only a certain fraction of the dose will reach the target site in the lung. Further fractions will be deposited in the inhalation device or the mouth-throat region. From the latter, deposited particles may subsequently be swallowed and absorbed in the GI tract, leading to potential parallel absorption processes to the systemic circulation (Fig. 1).

After being deposited in the lung drug particles have to dissolve, before pulmonary absorption into the systemic/ pulmonary circulation can take place. Besides absorption of dissolved drug, two competing pulmonary drug clearance processes may need consideration: pulmonary (metabolic) clearance, and pulmonary clearance processes consisting of mucociliary and macrophage clearance (12). Pulmonary dissolution, pulmonary absorption, and the lung-specific clearance processes (mucociliary and macrophage clearance) will be illustrated in the following sections and schematically in Fig. 1.

\section{Pulmonary Drug Dissolution}

Before absorption through the pulmonary epithelia can take place, inhaled drug particles that have been deposited in the conducting airways or the alveolar space of the lung have to dissolve in the pulmonary surface lining fluids. Pulmonary dissolution will be dependent on various drug (formulation) and physiological characteristics, e.g., lipophilicity or surface area of the inhaled drug/drug formulation, solubility of the drug in the airway lining fluids, and their volume and composition. Qualitatively and quantitatively, different compositions and volumes of the alveolar and the conducting airway lining fluids, as well as a thin fluid layer in the alveolar space (and in the peripheral conducting airways of the lung) might reason different dissolution characteristics dependent on where drug particles are deposited. However, it remains to be elucidated whether drug dissolution is faster in the peripheal or the central parts of the lung (13). Up to date quantitative characterization of the in vivo interplay of all contributing factors either with in vitro assays or with in silico methods is difficult. Especially for lipophilic drugs, pulmonary dissolution can represent the rate-limiting process and systemic exposure after drug inhalation is discussed to depend on the pulmonary drug dissolution characteristics (see below). A book chapter by Olsson et al. provides an excellent overview on pulmonary dissolution processes (13).

\section{Pulmonary Absorption of Dissolved Drug}

Drug inhalation is widely associated in literature with fast absorption of dissolved drug $(14,15)$. However, this often made association does not represent a generally applicable assumption. It is discussed that multiple absorption processes with different absorption rates might better represent pulmonary drug absorption. These absorption rates might differ for drug particles/droplets in different areas of the lung, e.g., between the alveolar space and the conducting airways. In the conducting airways, absorption of dissolved drug is often assumed to be slower compared with the alveolar space, due to less perfusion (16) and thicker airway walls (17). Conversely, absorption of dissolved drug in the alveolar space is often assumed to be fast due to a high local perfusion, a large absorption surface area $\left(100 \mathrm{~m}^{2}\right)$, and a thin diffusion barrier (12). In vitro, in vivo, and ex vivo animal assays have provided evidence for the proposed faster drug absorption in the alveolar space (18-20) compared with conducting airways.

\section{Pulmonary Mucociliary/Macrophage Clearance}

Drug clearance of drug particles/droplets from the lung depends on whether particles are deposited in the central or in the peripheral part of the lung as mucociliary clearance in the conducting airways is faster compared with the slow macrophage clearance $(21,22)$ in the alveolar space $(23)$. In addition, particles deposited in the central conducting airways are cleared faster than particles deposited in the peripheral conducting airways, as mucociliary clearance increases from 


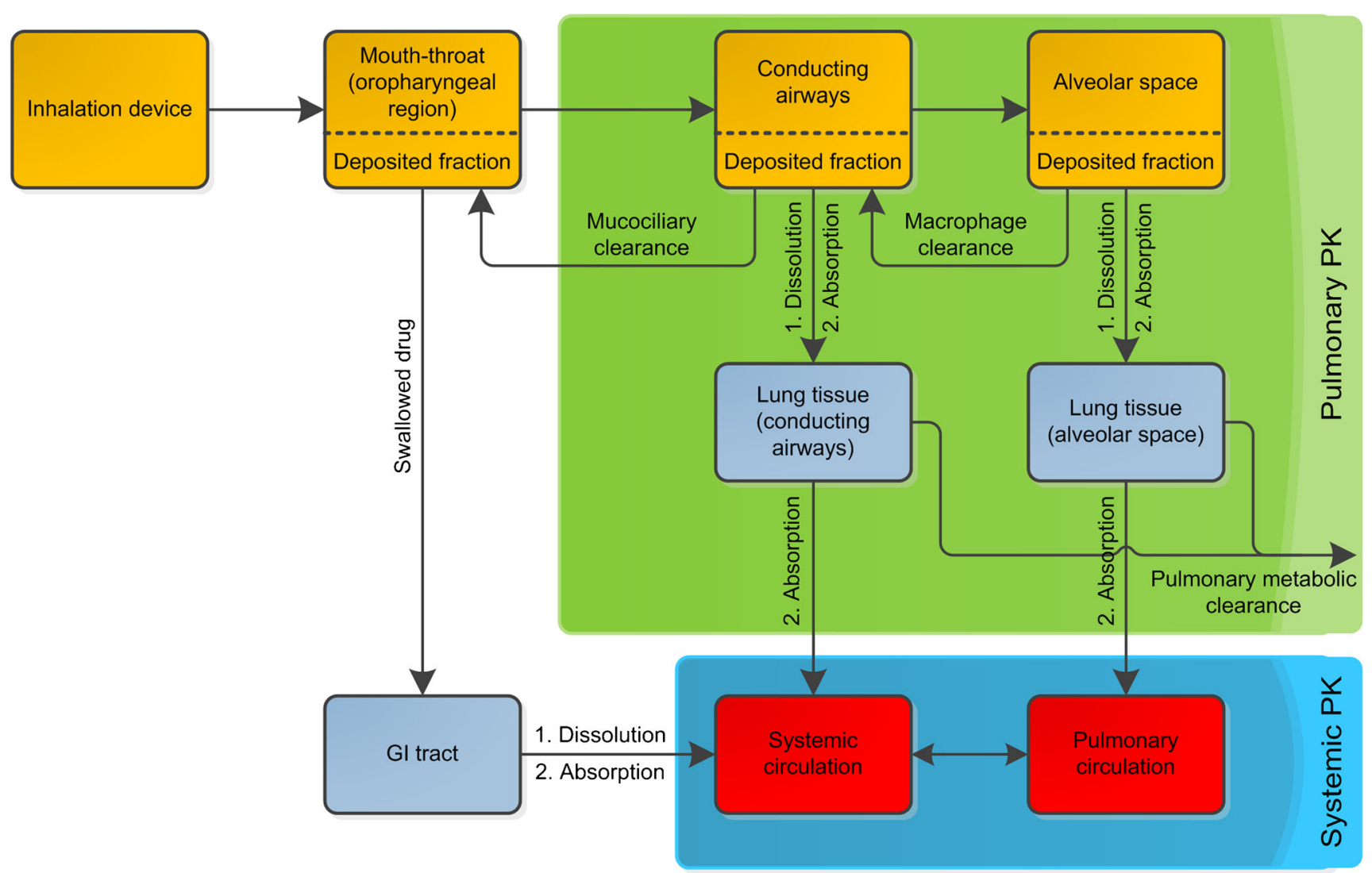

Fig. 1. Schematic representation of kinetic processes after drug inhalation. The three regions oropharyngeal, conducting airways, and alveolar space are kinetically distinct. Orange boxes, particle/droplet deposition compartments; light blue boxes, absorption compartments; red boxes, blood/plasma compartments; green area, processes associated with pulmonary PK; blue area, processes associated with the systemic PK. Here, the pulmonary absorption is represented as two-step absorption to the tissue, followed by absorption to systemic compartments; however, this process is often represented as a single step

peripheral (small bronchi) to central conducting airways (trachea). Mucociliary clearance clears particles in these airways to the mouth-throat region, where drug may subsequently be swallowed and absorbed from the GI tract (24). These pulmonary clearance processes are more relevant for undissolved drug particles (25), i.e., slowly dissolving drug formulations will be more susceptible than rapidly dissolving formulations or solutions. Consequently, the mucociliary clearance reduces pulmonary bioavailability of inhaled drugs. If oral bioavailability is lower compared with pulmonary bioavailability, mucociliary clearance also reduces the total bioavailability of inhaled drugs.

\section{Pulmonary Particle/Droplet Deposition Patterns}

The pulmonary deposition patterns describe the drug particle/droplet deposition in different areas of the lung (e.g., more pronounced drug deposition in the peripheral parts of the lungs or on a more detailed level more pronounced drug deposition in the alveolar space of the lungs) and might depend on multiple factors (see below). As outlined, these pulmonary particle deposition patterns are assumed to be relevant for both the pulmonary and systemic PK of inhaled drugs. Technological (e.g., formulation, device characteristics) and physiological aspects (e.g., airway geometry) as well as breathing patterns have been demonstrated to influence the deposition patterns and to be relevant for the PK of inhaled drugs; e.g., a larger aerodynamic particle diameter increases both the fraction deposited in the mouth-throat region and the fraction deposited in the central conducting airways (26). A more detailed review on the influence of particle size and the physical background on particle deposition has recently been published by Isaacs et al. (27); other technological aspects, e.g., the effect of drug formulations or different inhalers on pulmonary deposition, were reviewed by Labiris and Dolovich (28). Patient-specific factors such as age (29, 30 ), height (30), and sex (31) have also been demonstrated to influence particle deposition patterns and/or the PK after drug inhalation. Airway diseases (e.g., asthma bronchiale or COPD) and even different disease stages altered the particle deposition patterns (32): The total bioavailability of fluticasone propionate (33-35) was decreased in patients with more severe asthma bronchiale and COPD. Similarly, maximum plasma concentrations and the exposure were decreased for inhaled fluticasone propionate and budesonide after provoking bronchoconstriction (decreased forced expiratory volume in $1 \mathrm{~s}$ (FEV1)) in asthma patients (36). It was discussed that narrowed airways in patients (or after bronchoprovocation) would cause a more central pulmonary particle deposition compared with healthy volunteers (or before bronchoconstriction) and in combination with the more efficient mucociliary clearance in these central parts of 
the lung (see above) would result in a more efficient drug removal from the lungs. Finally, the handling of an inhalation device by the patient or even the capability of a patient to use the inhaler correctly (e.g., if reduced inhalation flow) and to hold the breath for a sufficient time span may impact the pulmonary deposition (37).

Even though the impact of technological, (patho-)physiological, and/or administration aspects on the (pulmonary) PK are qualitatively described in literature, a wide knowledge gap regarding the quantitative description of these relations remains. In particular, quantitative relationships between the pulmonary particle deposition patterns, the pulmonary absorption processes, and the drug clearance from the lung are (yet) lacking. Application of mathematical modeling approaches may offer opportunities for closing this knowledge gap.

\section{COMPREHENSIVE OVERVIEW ON MODELING APPROACHES FOR INHALED DRUGS}

Published modeling approaches describing the systemic (and pulmonary) PK of orally inhaled drugs can be categorized into two main groups: On the one hand, PBPK approaches, for which PK parameters are not estimated based on clinical data but derived from physico-chemical characteristics of the inhaled drug and on anatomical/ physiological characteristics. On the other hand, dataderived empirical PK approaches, for which PK parameters were estimated based on clinical PK data. Even if an empirical model contains a physiology-motivated model structure, these approaches will be referred to as empirical approaches, if all PK parameters were estimated based on clinical data. Both approaches can be further divided into several subcategories (Fig. 2). PK models, which cannot be categorized based on the above-described procedure, are considered as semi-empirical models.

\section{Technical Background of PBPK Models}

PBPK modeling is a mechanistic approach for predicting concentration-time profiles derived from the pulmonary PK (e.g., dissolution, absorption) and the systemic PK characteristics (e.g., distribution, excretion) by incorporating various anatomical and physiological aspects (e.g., organ perfusion rates or absorption surface areas) and drug/formulation characteristics (e.g., solubility, $\mathrm{p} K_{\mathrm{a}}$ values, partition coefficients, dissolution rates). In principle, a PBPK model is developed based on these predefined parameters without clinical data (bottom-up). To increase credibility and evaluate the descriptive performance of the PK model, an iterative process (learning and confirming) with predicting of and comparing with clinical data is recommended (38). PBPK models are often complex as they constitute a large system of (ordinary) differential equations containing all prior information and relevant mass transfer processes on a very detailed level (39).

\section{Technical Background of Empirical (Data-Derived) Models}

Compared with the PBPK approach, the empirical approach relies less on anatomical and physiological information but largely on clinical data (top-down). Pharmacostatistical methods and plausibility grounds (e.g., different pulmonary absorption rates based on physiological characteristics of the lung, see above) are determinants for developing a compartmental model that best describes the data. Empirical models are often less complex than PBPK models, typically have less mechanistic rationale and their PK parameters might be more difficult to interpret. Nonetheless, empirical models might also have a semi-mechanistic character, if prior information of the body ("system") or parts are included. Empirical modeling can be used to quantify the impact of individual characteristics (covariates) on PK parameters. Model evaluation may be performed with clinical data used for model building (internal evaluation) or additional clinical data (external evaluation) (40).

Two different empirical population data analysis approaches, the two-stage approach and the nonlinear mixedeffects modeling (NLME) approach, have been used to describe the PK after drug inhalation. In the two-stage approach, the PK model parameters are first estimated for each individual separately. In the second stage, summary statistics of the individual PK parameter estimates are calculated (40). In the NLME approach, all data is simultaneously analyzed, i.e., all population parameters including interindividual variability in these parameters are estimated

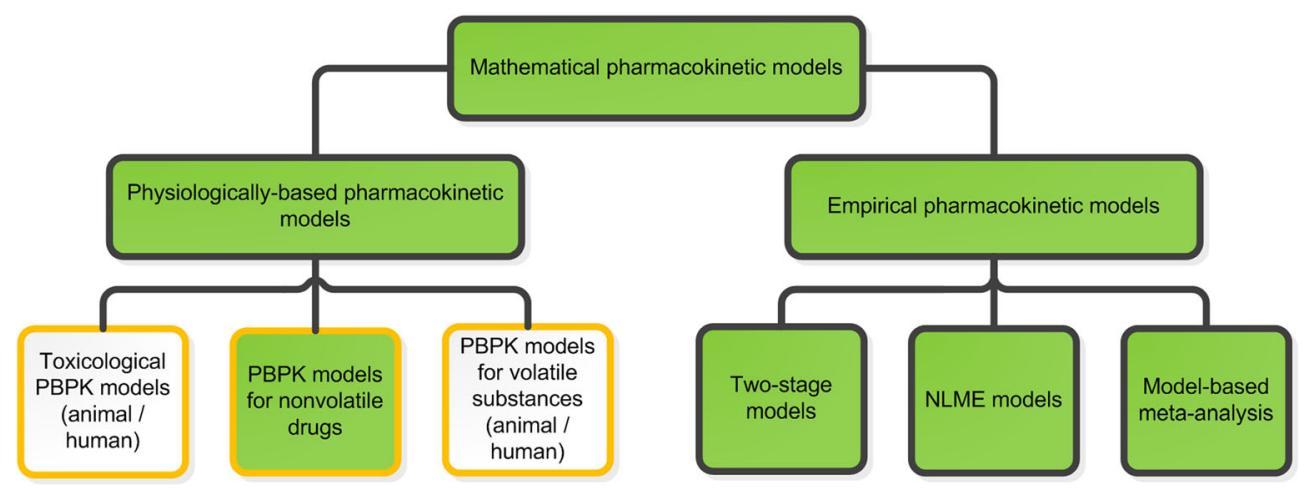

Fig. 2. Classification of mathematical PK models describing the pulmonary or systemic PK after substance/drug inhalation and model characteristics. Black boxes describe the type of mathematical approach; whereas orange boxes illustrate different aspects of the PK models. The filled boxes are further described and discussed in this review. NLME nonlinear mixed-effects, PBPK physiologically based pharmacokinetic, $P K$ pharmacokinetic 
in one step (40). Aside from these approaches, compartmental models have been reported being fitted to average plasma concentration-time profiles extracted from literature (modelbased meta-analysis) (41, 42). Non-compartmental approaches will not be addressed in this review.

\section{Comprehensive Overview on PBPK Models}

Published PBPK models describing the systemic/ pulmonary PK after inhalation of vapors were mostly used to assess the PK of inhaled anesthetics and other volatile substances (e.g., inhaled diacetyl (43)). A review about those PBPK models was published by Morris (44); underlying assumptions were homogeneous drug concentrations in the air and passive inhalation. However, these do not hold true for drug administration via an inhalation device representing an inhalation process with non-constant inhalation flow and non-uniform drug concentration in the inhaled air. Hence, these aspects should be considered in PBPK models of inhaled drug aerosols/ powders. Compared with the "vast mathematical descriptions of inhaled vapor disposition" (44), fewer PBPK models describing the human PK of orally inhaled drugs administered with an inhalation device have been published. These PBPK approaches will be reviewed in the following chapter categorized as drugspecific and generic (adaptable) approaches.

\section{Drug-Specific PBPK Models}

Gaz et al. published a theoretical study (not based on or evaluated with clinical PK data), in which a PBPK model was developed for predicting both the PK and PD of an inhaled bronchodilator (45). The nominal dose was divided into three fractions representing drug that was deposited inside the spacer/ inhalation device, in the mouth-throat region, and in the lung. The absorption process of the drug subsequently swallowed from the mouth-throat region was modeled as a separate absorption process from the GI tract into the systemic circulation. The pulmonary fraction was further differentiated into 17 fractions representing different airway generations (generation 0 represents the trachea, generation 16 the respiratory bronchioles). The locally deposited fractions were calculated by two mathematical functions: one described the deposition patterns of fine particles with pronounced deposition in airways, the other described the pronounced deposition of coarse particles in the mouth-throat region. These two functions were evaluated by comparison with simulated deposition patterns of inhaled particles calculated with the Multiple-Path Particle Dosimetry (MPPD; see Discussion) software program (46) showing an overall good agreement. A pulmonary dissolution process was not incorporated in the model as a droplet formulation was administered. After deposition in the lung, drug was quickly absorbed from the bronchial surface to the muscle compartments. The absorption rate constants of each airway generation to the specific muscle compartment and the transfer rate constant from the muscle to the plasma compartment were calculated for each of the 17 airway generations. The simulated PK results were not evaluated based on clinical PK data, but on PD predictions. Therefore, although PD is not the focus here, it is of high interest to shortly summarize, as the final PBPK model was evaluated based on efficacy predictions. For this purpose, bronchodilation in different airway generations was calculated based on drug amount in the corresponding muscle compartment of the airway generation, also assuming predefined airway obstruction patterns in asthma patients before drug inhalation. In a second step, the treatment-related lower airway obstructions were used to calculate the total airway resistance of all airways. Finally, the total airway resistance was used to calculate FEV1 over time and compare the result to reported FEV1 profiles (47). Even though this model has only been evaluated for one bronchodilator, Gaz et al. underlined that this model might be adapted for different bronchodilators or drug formulations, e.g., for different inhaled particle sizes.

\section{Generic PBPK Models}

Generic PBPK models for inhalation have recently been presented being implemented in software programs. Hence, they can only be outlined in the context of the software tools: For GastroPlus ${ }^{\mathrm{TM}}$, the Additional Dosage Routes Module (ADRM ${ }^{\mathrm{TM}}$ ) expands the classical implemented PBPK model to the specific characteristics of drug absorption after inhalation (48). Pfizer's developed in-house model has been embedded into the PulmoSim ${ }^{\mathrm{TM}}$ software (49). These two models will be referred to as 'model GP' and 'model PS', respectively.

Model GP uses an approach analogous to that by Gaz et al. (45). As a first step, the pulmonary deposition patterns of particles have to be specified by choosing between the two following options: If prior information on pulmonary particle deposition patterns is available, the deposition patterns can be defined manually. Alternatively, if deposition patterns are unknown, the software allows their calculation by means of an embedded particle deposition model published by the International Commission on Radiological Protection (ICRP 66 model) (17). Both the model GP and the ICRP 66 model divide the airways into four different pulmonary compartments (Fig. 3), namely the extra-thoracic, thoracic, bronchiolar, and alveolar-interstitial compartment (17). Particles deposited in the extra-thoracic compartment are swallowed and GI absorption is calculated with an Advanced Compartmental Absorption and Transit (ACAT) absorption model (50). The dissolution process of particles that are deposited in one of the three remaining pulmonary compartments is calculated with e.g., the Noyes-Whitney function (51) including various particle and mucus characteristics (e.g., $\mathrm{pH}$ value).

Pulmonary absorption to the plasma of dissolved drug is considered as a passive process that is specifically calculated for each of the three pulmonary absorption compartments by accounting for both human pulmonary anatomical and physiological parameters (e.g., surface area, thickness and volume for mucus and pulmonary cells) and drug-dependent input parameters (e.g., pulmonary permeability) (48). Other aspects of drug inhalation such as different mucociliary clearances depending on the specific pulmonary compartment, pulmonary metabolism, or mucus binding of the drug are included in the model GP. However, as an adequate distribution of metabolizing enzymes or transporters and their transformation kinetics are rarely found in literature, the model GP may still need adaption for each specific research question. The PBPK model was evaluated by simulating and comparing the plasma concentration-time profiles of inhaled drugs with clinical data, e.g., morphine (52), triamcinolone acetonide (53), budesonide (54), and tobramycin (55). While 


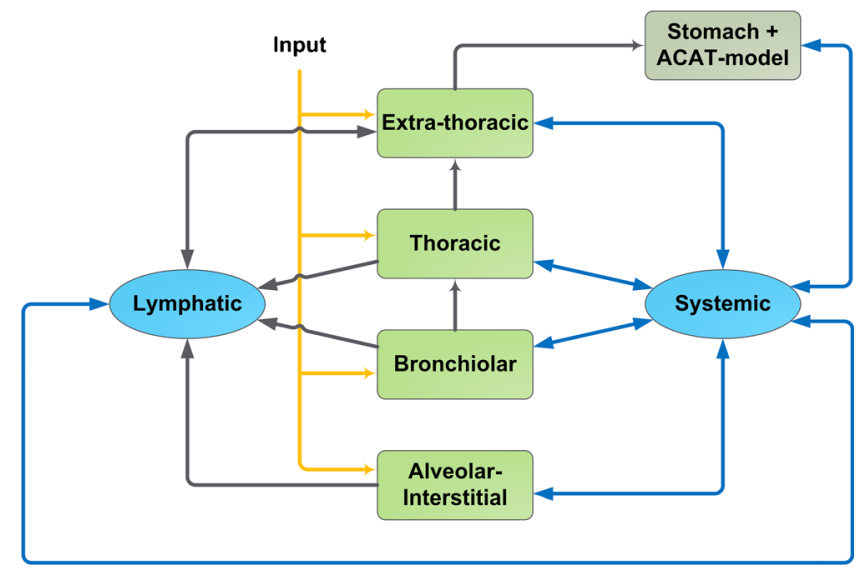

Fig. 3. PBPK absorption model for inhaled drugs (Additional Dosage Routes Module implemented in the GastroPlus ${ }^{\mathrm{TM}}$ software). Yellow arrows, drug input to the lung compartments; light green boxes, lung compartments; dark green box, GI tract compartment (stomach, Advanced Compartmental Absorption and Transit model); blue boxes, systemic disposition compartments; black arrows, transport processes to/from the lung; blue arrows, systemic distribution processes, adapted and modified with permission from (48)

some PK parameters were fitted based on clinical data, it was emphasized that concentrations for inhaled budesonide were simulated without fitting parameters to measured concentrations. It was concluded in that article that the predictions provided a "very reasonable agreement" (54) to observed concentrations.

The second generic PBPK model for inhaled drugs, namely model PS, differentiates the inhaled drug into two separate fractions to adequately predict the absorption characteristics (Fig. 4) (49). One fraction is deposited in the lung, whereas the remaining fraction is absorbed in the GI tract. Particles in the lung may dissolve and dissolved drug is either absorbed to lung tissue or cleared by the mucociliary clearance to the gut compartment before absorption takes place. Both undissolved and dissolved drug is cleared unless it has been absorbed into lung tissue. Absorption of drug from the pulmonary tissue to plasma is dependent on the unbound fraction of the drug in the lung meaning that a high tissue affinity of drug in the lung results in both high pulmonary retention/transition times and slow pulmonary absorption to plasma. Unfortunately, to this date, there has been no publication using this PBPK model so that it is not possible to give more detailed information on this model and, more importantly, to assess its performance.

Aside from these models, other generic PBPK models are available; however, (yet) without a specific submodel to account for the absorption characteristics of oral drug inhalation, e.g., the SimCyp Simulator ${ }^{\mathrm{TM}}$ describes the pulmonary absorption of drug with a single first-order absorption process (56). These PBPK models will not be further discussed here.

\section{Comprehensive Overview on Empirical (Data-Derived) PK Models}

Compared with the previously discussed PBPK approaches, more publications described the systemic PK after drug inhalation with empirical models. A full list of empirical models and the corresponding model structure can be found in Table I.
The structure of most absorption models in literature was comparable and can be attributed to one of the two main absorption model types, model type I and model type II, as illustrated in Fig. 5. The majority of empirical PK models was based on inhalation data alone and described the systemic PK by a one or two compartment systemic disposition model with a single absorption process (model type I, Fig. 5). When additional data was available (e.g., IV, subcutaneous (SC), oral), the absorption models tended to increase in complexity. These more complex PK models often described the absorption with parallel absorption processes describing pulmonary and GI tract absorption processes (model type II, Fig. 5). In general, if drug was bioavailable after swallowing, the absorption from the GI tract was described by a single absorption process, whereas pulmonary absorption was described with multiple absorption processes (57-60). Integration of additional aspects in the model types (e.g., pulmonary clearance processes) led to the model subtypes presented in Fig. 5.

\section{Empirical PK Models Based on Inhalation Data Only}

When no IV or other additional data was available for model development, the model type I was preferentially used to describe the PK of inhaled drugs. For instance, empirical models describing the PK of inhaled corticosteroids (ICS), namely ciclesonide (and its active metabolite) (61-63), budesonide (64, $65)$, fluticasone proprionate $(63,65-67)$ implemented a single absorption process. Other models based only on inhalation data described the PK of albuterol (68, 69), formoterol (70), indacaterol (71), PF-00610355, $\beta_{2}$-adrenoceptor agonist, (72), zanamivir (73), laninamivir octanoate, prodrug transformed in the lung, and its active form laninamivir (74), GLP-1 (75), tobramycin (76), and HMR1031, VLA-4 antagonist (77) characterized the absorption by the same models. The single absorption process was implemented as a first-order (62-66, 68, $73-77)$ or a zero-order absorption process $(67,69)$. Variations of model type I included the absorption process being implemented as an IV bolus input or as a fixed high first-order absorption rate constant $(62,65)$, as the available PK data did not allow 


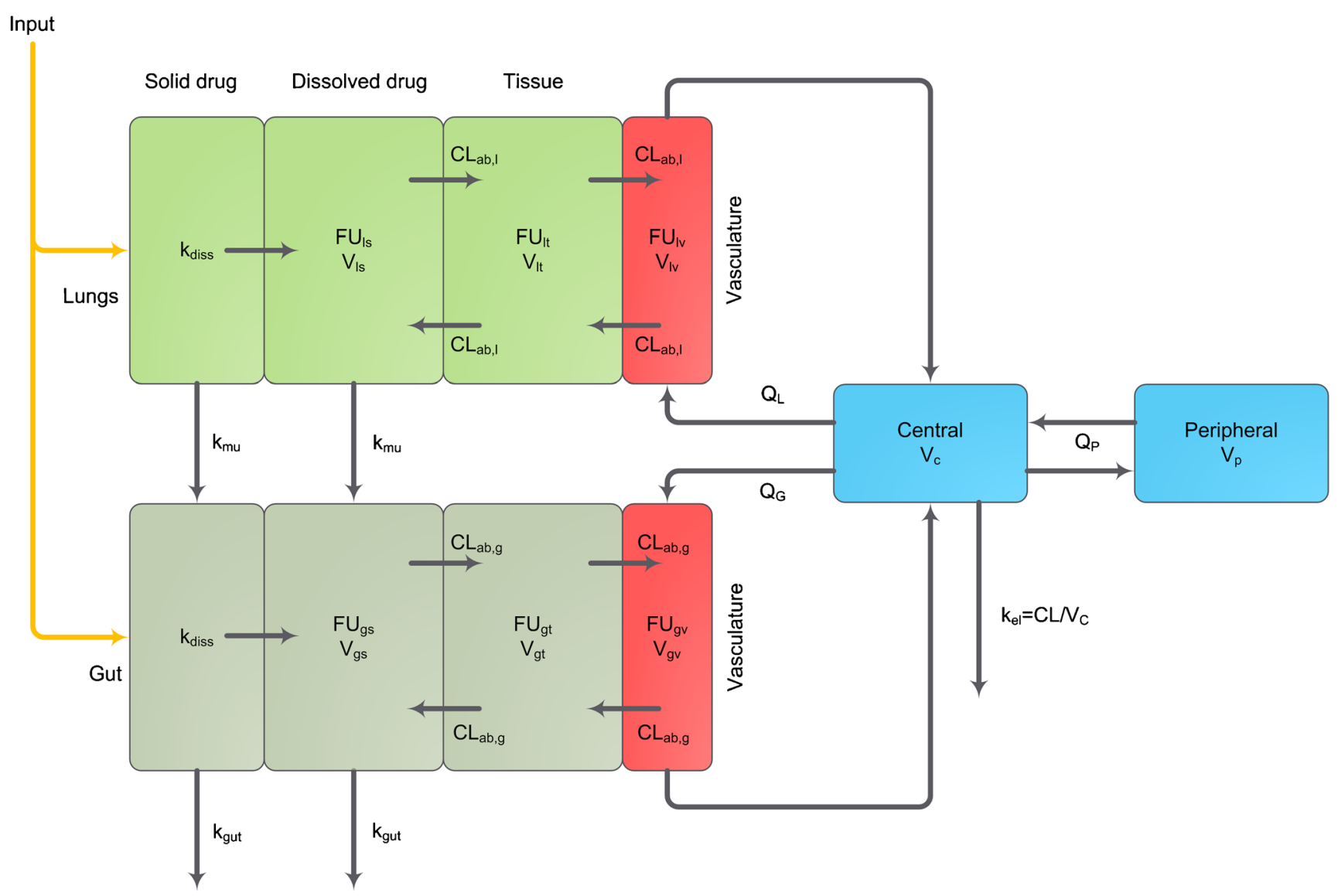

Fig. 4. PBPK model for inhaled drugs (PulmoSim ${ }^{\mathrm{TM}}$ ). Subscripts: $l$ lung, $g$ gut, $s$ solution, $t$ tissue, $v$ vasculature; parameters: $k_{\text {diss }}$ dissolution rate constant, $k_{m u}$ mucociliary rate constant, $k_{\text {gut }}$ elimination rate constant from the gut, $k_{e l}$ elimination rate constant from the central compartment, $F U$ fraction unbound, $C L_{a b}$ absorptive clearance across tissue, $V$ physiological distribution volumes, $Q_{G}$ flow rate to the gut vasculature, $Q_{L}$ flow rate to the pulmonary vasculature, $Q_{P}$ intercompartmental clearance; light green boxes, pulmonary compartments; dark green boxes, gut compartments; red boxes, vasculature compartments in the specific organs; blue boxes, systemic disposition compartments

estimating the high absorption rate constant $(71,74)$. Alternatively, the use of a transit absorption model was described (model subtype I-a, Fig. 5) (72). Even though only inhalation data was available, PK after formoterol inhalation was characterized more detailed by model type II (70) with one pulmonary and one absorption process from the GI tract due to a measurable double peak in the plasma concentration-time profile and assuming significant oral bioavailability based on prior information.

\section{Empirical PK Models Based on Inhalation and Additional Data}

When IV data was additionally available for model development, the complexity of the resulting PK models increased. Especially the absorption model tended to be more detailed, whereas the majority of the systemic disposition models remained the same. For instance, the absorption process of morphine was described by a two compartment absorption model (60), whereas the absorption of olodaterol (57) and glycopyrronium (59) was described by three parallel pulmonary absorption processes characterized with different absorption rate constants (see model type II, Fig. 5). GI tract absorption was either negligible or modeled separately from pulmonary absorption. However, for this purpose additional oral data or inhalation data with charcoal was necessary. By administering charcoal in addition to inhaled glycopyrronium to prevent GI tract absorption of swallowed drug, Bartels et al. (59) were able to differentiate between pulmonary and GI tract absorption. An even more complex version of model type II was identified for prochlorperazine, as the absorption from an absorption compartment was characterized with three parallel transit compartments. However, differing from the other models for drug inhalation, the inhaled dose as well as the IV dose were administered into the absorption compartments (model subtype II-a, Fig. 5) (78).

Moreover, by including IV or subcutaneous data into the empirical analysis, three groups characterized the PK of insulin with a first-order absorption process (model type I, Fig. 5) (41, 42, 79), whereas Sakagami described the firstorder absorption process parallel to a pulmonary first-order loss that was discussed to represent the pulmonary metabolic degradation of insulin (model subtype I-b, Fig. 5) (42).

\section{Semi-Empirical Models}

Besides the discussed empirical approaches, Stass et al. presented a more mechanistic modeling approach to describe 


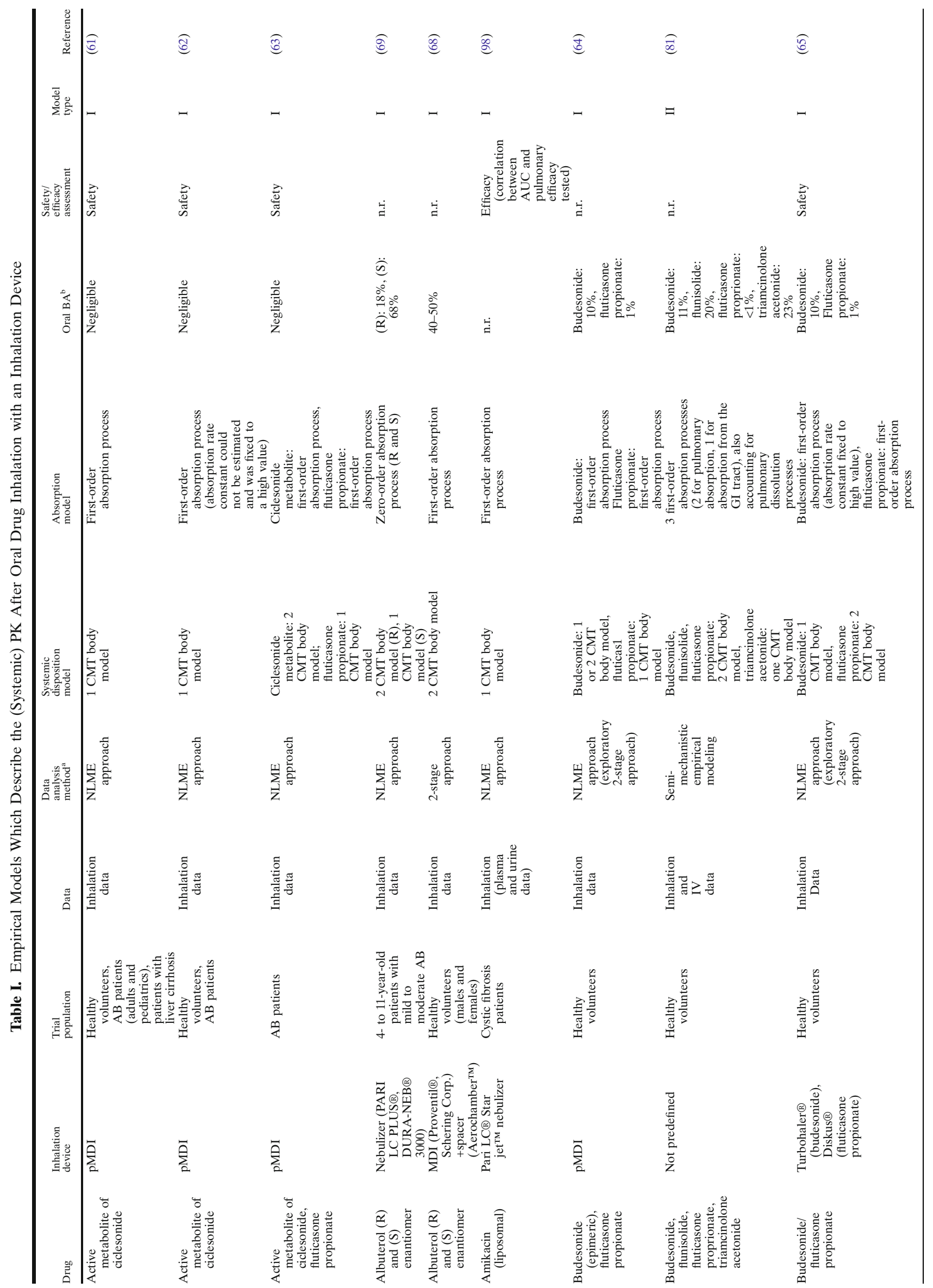




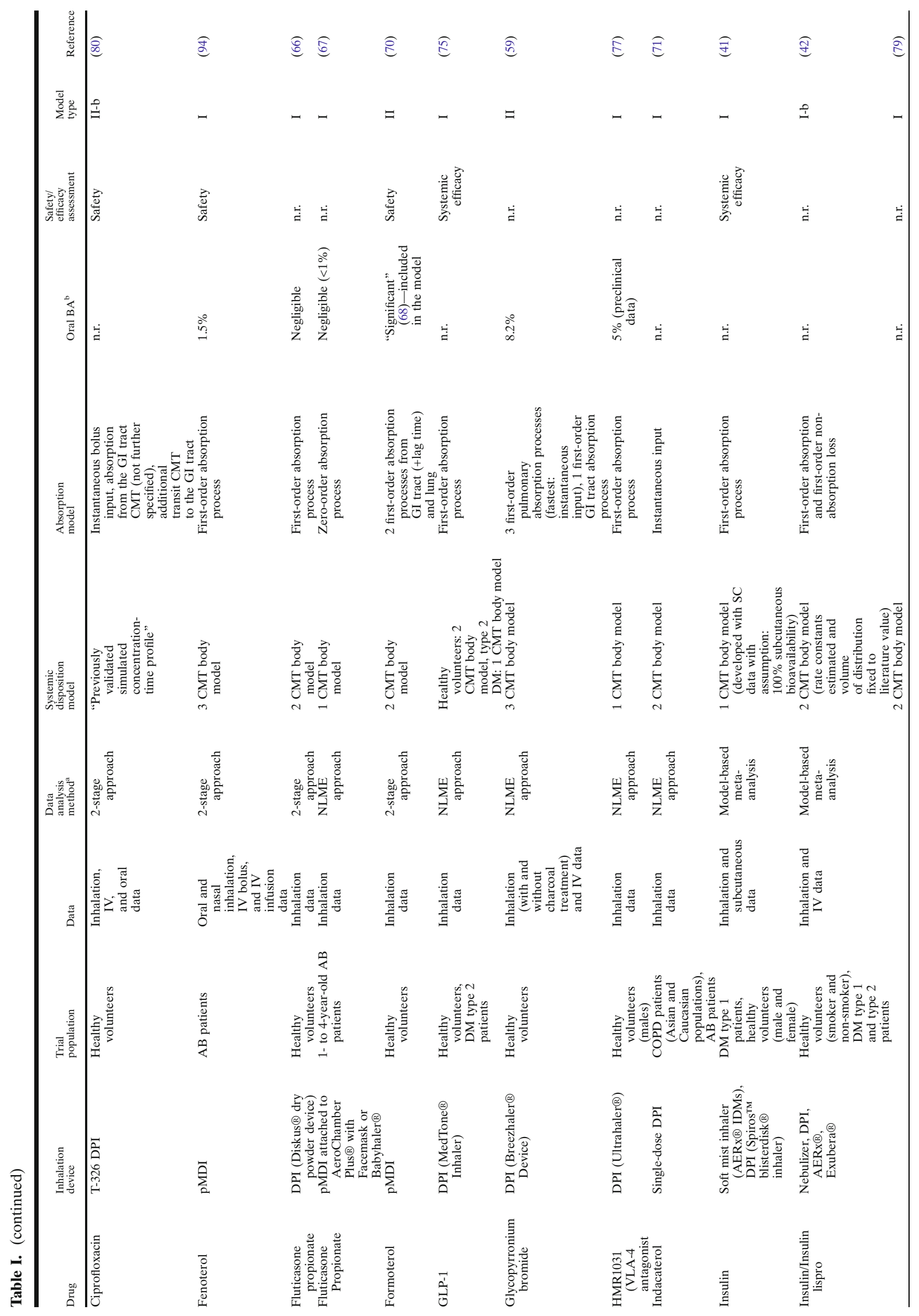




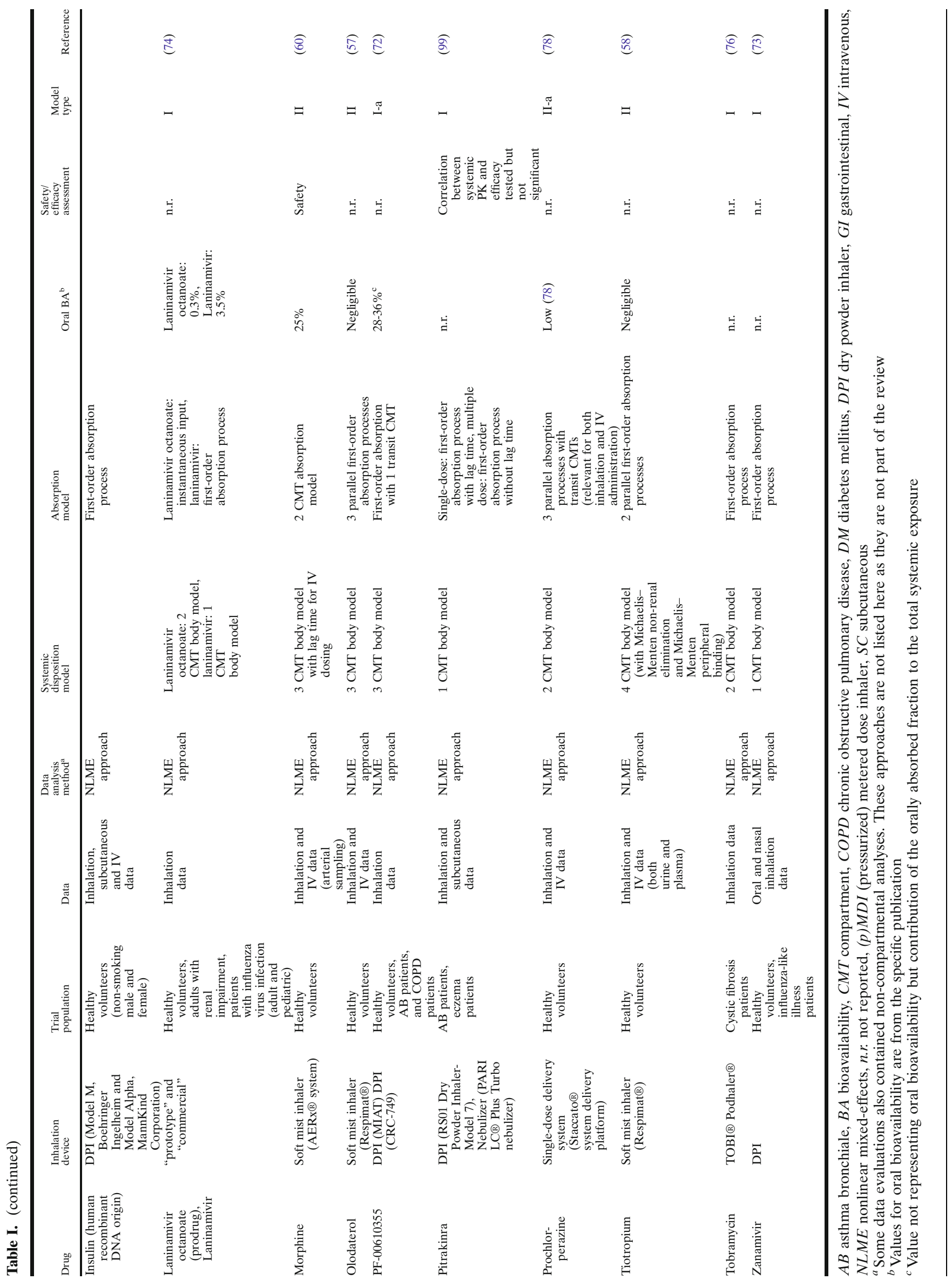



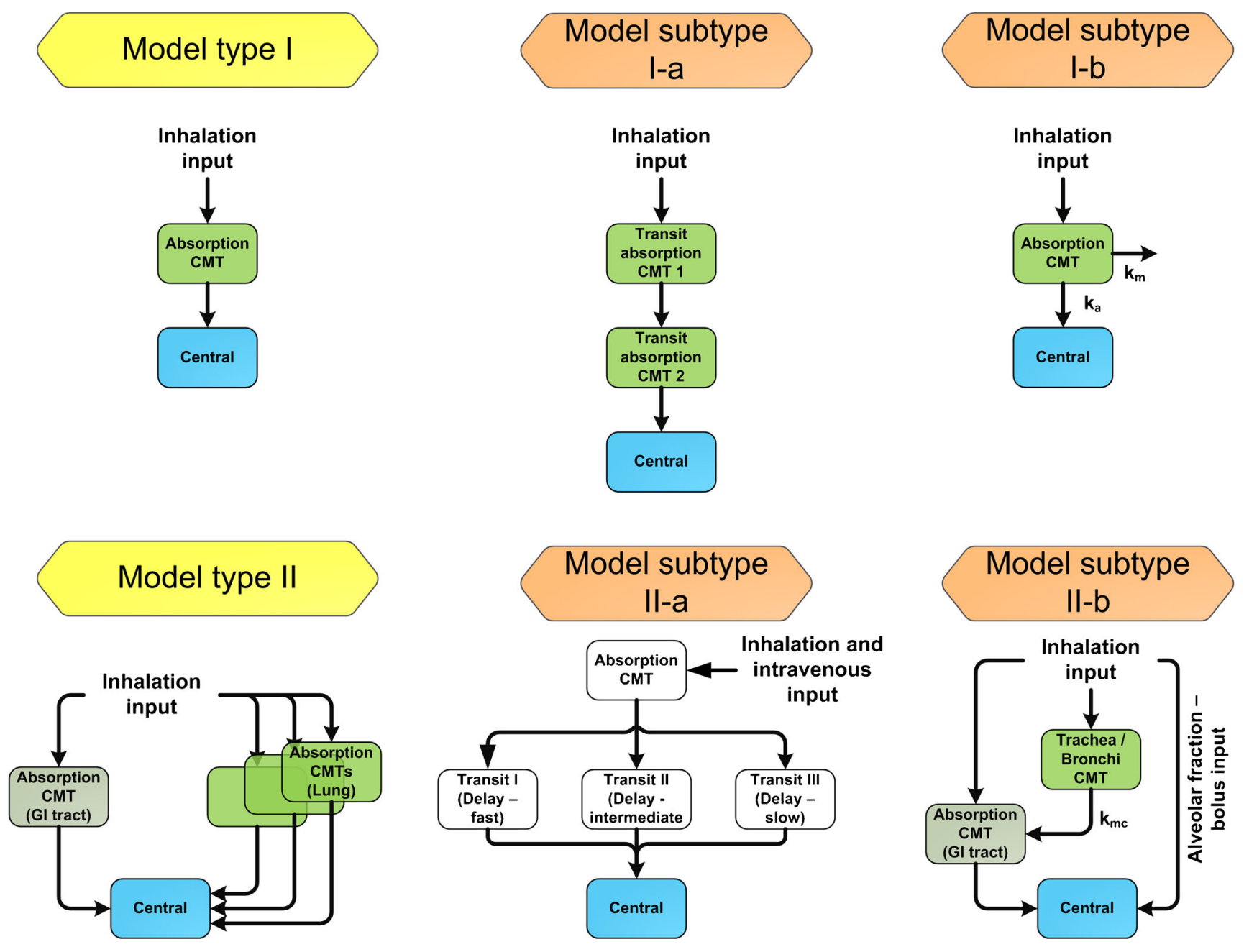

Fig. 5. Absorption model types (yellow boxes) and model subtypes (orange boxes) used for empirical PK modeling. Only structural models that were published and applied for modeling of plasma concentration-time profiles after oral drug inhalation are presented. Model type I, single absorption compartment linked to a systemic disposition model; model subtype I- $a$, absorption with a transit compartment model; model subtype I-b, absorption with parallel pulmonary metabolism; model type II, multiple absorption compartments linked to a systemic disposition model; model subtype II- $a$, absorption with three parallel transit absorption compartment models; model subtype II-b, absorption model with different absorption processes: absorption in the alveolar space treated as pulmonary bolus input (instantaneous absorption), transit absorption compartment (trachea/bronchi), and GI tract absorption. $k_{a}$ absorption rate constant, $k_{m}$ metabolism rate constant, $k_{m c}$ mucociliary rate constant; light green boxes, pulmonary compartments; dark green boxes, GI tract compartments; blue boxes, systemic disposition model. For convenience, the systemic disposition model is simplified to a single central compartment

the PK of ciprofloxacin (80) and Weber et al. published an approach in which the PK parameters were not estimated based on clinical data, but derived from literature (81). To describe the PK of ciprofloxacin, the model was based on prior knowledge about the PK of inhaled drugs (model subtype II-b, Fig. 5). It was assumed that the fraction deposited in the alveolar space $\left(\mathrm{F}_{\mathrm{Al}}\right)$ was instantaneously absorbed to the plasma, whereas the fraction deposited in the mouth-throat $\left(\mathrm{F}_{\mathrm{MT}}\right)$ was completely swallowed and absorbed in the GI tract. No absorption in the trachea and bronchi was "allowed" so that drug deposited in the trachea/bronchi $\left(\mathrm{F}_{\mathrm{TB}}\right)$ was assumed to be completely transported by the mucociliary clearance (characterized with $\mathrm{k}_{\mathrm{MC}}$ ) to the mouth-throat and subsequently swallowed. However, the PK parameters describing the absorption $\left(\mathrm{F}_{\mathrm{Al}}, \mathrm{F}_{\mathrm{MT}}, \mathrm{F}_{\mathrm{TB}}, \mathrm{k}_{\mathrm{MC}}\right)$ were not based on, e.g., prior deposition information but estimated by a two- stage approach. Hence, this PK model is considered as an empirical model.

The PK model by Weber et al. was developed on the basis of a previously published "theoretical PK/PD model" (10). This PK/PD model was extended with PK parameter estimates from the literature (e.g., the PK parameters of the systemic disposition model) for inhaled corticosteroids (81). PK parameters not available from literature, such as for absorption characteristics had to be assumed (absorption rate constants, pulmonary deposition patterns). The mouth-throat fraction was assumed to be swallowed and dependent on the defined oral bioavailability, might be absorbed in the GI tract. The pulmonary dose (drug deposited in the lung) was further differentiated into two kinetically differently absorbed fractions (central/peripheral). In addition, mucociliary clearance and dissolution rate estimates were derived from literature 
and included in the PK model. Simulations performed with the PK model were compared with the PK results of four studies and indicated a satisfactory predictive performance of the PK model.

\section{Covariates in Empirical PK Models}

Besides the structural part of empirical PK models, which describes the typical behavior in absorption and systemic disposition after drug inhalation (see Fig. 5), covariates (patient-specific characteristics) were often implemented in the PK models to explain variability in the PK parameters between different individuals or population subgroups. Covariates identified to explain interindividual variability of absorption parameters (absorption rate constants, pulmonary bioavailability, etc.) were (i) cigarette smoking and (ii) deeper inhalation; both predictors increased the pulmonary absorption rate constant and the pulmonary non-absorptive loss of insulin (42), (iii) a higher inhaled insulin dose that was demonstrated to decrease the pulmonary bioavailability, but increase the pulmonary absorption rate constant (41), (iv) the disease status: diabetes mellitus type 2 increased the pulmonary absorption rate constants (75), whereas asthma patients had a higher pulmonary absorption rate constant and a lower relative bioavailability (72), and (v) different inhalation devices (72).

\section{DISCUSSION}

\section{Physiologically Based Pharmacokinetic Modeling}

Depending on drug-specific characteristics, different aspects might need consideration in the modeling approach; e.g., if a particle formulation is administered and the drug/ drug formulation is slowly dissolving in airway fluids, literature suggests that both the dissolution process and the pulmonary clearance processes are of kinetic relevance for modeling both the systemic and pulmonary PK. The PK predictions of the PBPK model do not only depend on the choice of implemented aspects (e.g., if a pulmonary drug dissolution is implemented into the model), but also on their way of inclusion in the model (e.g., how pulmonary dissolution is characterized). The main aspects that were implemented in the PBPK models will therefore be discussed in the following sections; however, not as a complete list of all possibly relevant aspects-additional aspects such as pulmonary metabolism may additionally need consideration.

\section{Pulmonary Drug Deposition Patterns}

Both Gaz et al. (45) and the model GP (48) identified the pulmonary particle deposition patterns as a relevant factor contributing to both the pulmonary and systemic PK. The model GP implemented the ICRP66 model to predict pulmonary drug deposition, whereas Gaz et al. evaluated the deposition patterns with the MPPD model. Both the MPPD and the ICRP 66 deposition models originate from the toxicology area and were developed with healthy volunteer data (17); these deposition models, therefore, only partly reflect deposition characteristics after drug inhalation with an inhalation device. Thus, using the ICRP 66 model for predicting deposition patterns (in patients) after drug inhalation with a metered dose inhaler (MDI) or dry powder inhaler (DPI) led to overprediction of the pulmonary dose and underprediction of the mouth-throat fraction (82). Possible explanations for the constantly overpredicted pulmonary dose might be an inhaler spray momentum (ballistic effect; high aerosol velocity produced) of MDIs (83), turbulent inhaler jet effects (84), or coarse effects (particle deagglomeration dependent on inhalation flow) of DPIs (28, 85). The lung deposition of a nebulizer without a strong ballistic or coarse effect was accurately predicted (82).

Computational Fluid Dynamics models (reviewed by Longest and Holbrook) (86) could offer a solution to overcome the illustrated drawbacks of currently used particle deposition models. To this date, they are however computationally too intensive (86) for applying them for standard PBPK analyses.

\section{Dissolution and Pulmonary Clearance Processes}

The model by Gaz et al. (45) did not include any dissolution step. Therefore it is not adaptable to situations in which pulmonary dissolution is of importance. This might be relevant even for inhaled solutions as drug may still deposit (after evaporation processes and crystallization of the drug) in form of solid drug particles. Currently, little quantitative information is available about pulmonary drug dissolution. Additional knowledge is necessary for realistically including pulmonary dissolution in a PBPK model, e.g., how to quantify pulmonary dissolution in different areas of the lung, as differences in both composition and volume of the airways lining fluid exist dependent on where drug is deposited $(12,13)$. A non-uniform pulmonary deposition of particles may theoretically further impact the dissolution process, e.g., particle agglomerates at airway bifurcations might dissolve more slowly compared with single uniformly deposited particles. This example underlines importance of in vitro assays that adequately represent pulmonary specific dissolution characteristics. In general, in vitro in vivo correlations (IVIVC) need to be demonstrated before PBPK approaches can be used to predict reasonably both pulmonary (regional) and systemic PK of inhaled drugs.

An adequate characterization of the pulmonary dissolution process might even be more important, as undissolved particles are cleared by the pulmonary clearance processes. Both model PS and the model GP account for mucociliary clearance processes that "back-transport" the drug to the mouth-throat region. A second, slower process that may represent the macrophage clearance from the alveolar space was not included. However, this simplification may be appropriate as this clearance process with a half-life of at least $\sim 35$ or $\sim 115$ days (reported by ICRP (17) and NCRP (87), respectively) should not be relevant for drug particles administered by inhalation.

\section{Pulmonary Tissue Affinity of Drugs}

Model PS additionally underlined the importance of pulmonary (subcompartmental) tissue affinity for some inhaled drugs. For many drugs, especially weakly basic amines, a high affinity to the lung tissue was discussed, possibly due to 
lysosomal trapping (88). Alternatively, binding effects, such as target binding to the receptor (89) or exosite binding (binding to a location near the receptor that allows the drug to angle on and off the receptor, discussed for salmeterol (90)) were discussed to explain either a long pulmonary residence time of a drug or/and the long efficacy of inhaled drugs. Both slow drug-receptor dissociation as well as binding to an exosite as rate-limiting processes might delay absorption to systemic disposition and therefore can contribute to long lasting efficacy after drug inhalation. As mentioned for pulmonary dissolution and pulmonary absorption of dissolved drug the impact of these "distribution effects" may depend on the inhaled drug characteristics. If kinetically relevant these effects will be important to consider as they might represent the reason for apparent flip-flop kinetics after drug inhalation. It should also be emphasized that pulmonary distribution processes are relevant after both IV and inhalational drug administration. Redistribution from the systemic drug disposition to the specific model compartments, which represent these "distribution processes," should be accounted for (see Model PS, Fig. 4).

\section{Summary}

In summary, different PBPK approaches have been reviewed, concerning how to mechanistically model and thus simulate the PK of inhaled drugs. Depending on the purpose of the PBPK model (generic or drug-specific), pulmonary dissolution was included in the PBPK model. Other potentially relevant pulmonary $\mathrm{PK}$ characteristics were not covered, even by the generic PBPK models, e.g., enzyme or transporter distribution across the lung and their activity or the influence of smoking on the airway characteristics. Therefore, individual airway characteristics in healthy volunteers (or even more pronounced in patients) and the resulting impact on the systemic (and pulmonary) PK may not have been adequately captured by these PBPK models. To account for those limitations, a sound understanding on all abovedescribed relevant PK aspects, such as particle deposition, pulmonary dissolution, absorption of dissolved drug and the interplay of these processes is crucial. As a first step, demonstrating IVIVC or in silico in vivo correlation is necessary before reasonable implementation into (generic) PBPK models. Implementing in vitro data as input for the PBPK models (after demonstrating IVIVC) might also enable to implement population characteristics, e.g., predict the impact of different inhalation flows on lung deposition (example of IVIVC demonstration by Olsson et al. (91)), even in disease populations such as patients with asthma bronchiale or COPD. After demonstration of IVIVC and implementation of the above described aspects will be accomplished, more model evaluation will be necessary with additional clinical PK data of inhaled drugs to demonstrate adequateness of model-based simulations.

\section{Empirical (Data-Derived) PK Modeling}

The availability and quality of clinical data are crucial aspects for building an appropriate PK model after oral drug inhalation. Without the availability of IV data (which is frequently not-publicly-available), it is impossible to determine the actual systemic clearance and volume of distribution parameter value (i.e., both can only be determined as apparent parameters, i.e., as a function of the systemic bioavailability). Moreover, drawing conclusions based on inhalation data alone about absorption or dissolution characteristics of the inhaled drug, i.e., the occurrence of the often mentioned flip-flop kinetics after drug inhalation $(42,66,92)$ is not feasible. These identifiability issues are also relevant for orally administered solid dosage forms. For inhaled drugs, however, the situation is even more pronounced as the drug can reach the systemic circulation via both routes, the lung and GI tract. Thus, data after IV and oral administration as well as inhalation is highly desirable to allow determination of all PK parameters and specifying the pulmonary drug absorption appropriately. The challenge of flip-flop kinetics after drug inhalation has been discussed in more detail in (66).

\section{Data Below the Lower Limit of Quantification}

Even though the lower limit of quantification (LLOQ) of assays measuring drug concentrations have decreased over the last decades, another data-specific issue after drug inhalation might be the occurrence of plasma concentrations below the LLOQ, as a result of low administered doses for inhaled drugs. Ting et al. discussed this topic for tobramycin, but concluded that for their analysis the low fraction of values below the LLOQ $(6.8 \%)$ in their dataset could be ignored during model development (76). However, this should not be a general rule, and it is recommended to consider data below the LLOQ in the modeling approach, especially if the censored concentrations represent a considerable fraction in the dataset (93). For tiotropium (58) with a high fraction of data below the LLOQ, urine data were additionally evaluated to adequately characterize the terminal phase. In comparison with plasma data, concentrations in urine might be above the LLOQ for a longer time span (if the drug is renally excreted). Hence, urine data might provide a good alternative or addition to accounting for plasma data below the LLOQ.

\section{Empirical PK Models Based on Inhalation Data Only}

Estimation of absorption rate constants was often not feasible. Alternatively, characterizing the absorption as zeroorder or bolus input accounted for the lack of data in the absorption phase. It might be emphasized that a first-order absorption rate constant is physiologically more plausible, as a pulmonary zero-order absorption rate constant would only be justified if drug is constantly administered over time (e.g., constant drug nebulization). Dependent on the purpose of the PK analysis (e.g., assessing systemic pharmacodynamics), a single absorption rate constant might be sufficient to describe the (systemic) PK after drug inhalation. However, a more detailed characterization of the absorption processes would be desirable if the purpose of the analysis is to extrapolate to unstudied settings, to increase the mechanistic understanding or to assess local pulmonary efficacy. Compared with oral dosing, the pulmonary absorption process may start directly after drug inhalation (without a lag time); without an adapted sampling scheme the first measured concentration may already represent the $C_{\max }$. More extensive sampling in the 
absorption phase could accommodate this issue. Thereby, it might be possible to differentiate oral from pulmonary absorption, even without IV data (due to a second $C_{\max }$, see formoterol example (70)).

\section{Empirical PK Models Based on Inhalation and Additional Data}

Additional data of different administration routes allowed investigation of the absorption specific aspects independent of systemic drug disposition. For instance, Bartels et al. were able to identify a very slow pulmonary absorption process for glycopyrronium (59): Systemic PK parameters (e.g., CL, V) had been estimated with empirical modeling of IV data beforehand and were treated as fixed parameters during further model development. Otherwise, the very long terminal half-life and the accumulation of the drug could also have been explained by an additional body compartment with very slow drug kinetics. However, by fixing the systemic disposition parameters it was (reasonably) assumed, that the healthy individuals in both trials (IV and inhalation trial) were comparable. Nonetheless, most IV data is only available in healthy volunteers, and the population characteristics (age, creatinine clearance, etc.) between (elderly) COPD patients and healthy volunteers typically differ. This may be one hurdle for developing empirical models in diseased populations (only one publication provided fenoterol IV data of asthma bronchiale patients) (94). Nevertheless, the approach of fixing the systemic disposition parameters still seems to be the most reasonable method and was extensively used if IV data was available $(42,57,59,60)$. Alternatively, Potocka et al. (79) and Avram et al. (78) estimated the systemic disposition and inhalation PK parameters simultaneously. This approach may have the disadvantage, that an inappropriate absorption model could bias the systemic PK parameters and vice versa. However, further investigation by direct comparison of the results of both approaches is advocated.

Even though the PK models based on both inhalation and IV data tended to support more complex characterizations of the absorption processes, one single absorption process was also demonstrated to appropriately describe the PK of some inhaled drugs. One explanation might be that while the absorption processes through the different barriers of the different airways may differentiate, the dissolution of the drug particles at different sites in the lung may not. Hence, in addition to the slower dissolution process, the more lipophilic character of, e.g., inhaled corticosteroids compared with other drugs, may result in a faster diffusion through the epithelial barriers of the lung (95). A slow dissolution process in the pulmonary fluids in combination with a fast absorption process of the dissolved drug to the plasma might then appear as a single absorption process. Based on clinical data alone, it might not be possible to differentiate between the dissolution and the absorption process, and to this date including information from in vitro dissolution assays may not be trivial as no versatile in vitro dissolution assay representing pulmonary dissolution processes has been established (95-97). Another physiologically plausible explanation for only a single absorption process might be relevant for insulin. Insulin is only negligibly bioavailable after oral dosing and the models implemented pulmonary metabolism of insulin $(41,42)$. This feature could explain why no slower absorption process of insulin was apparent and only one absorption process was identified that might represent absorption in the alveolar space.

Most empirical PK models developed on both inhalation and IV data indicated at least two absorption processes differing in their absorption half-lives and the corresponding fractions absorbed necessary to describe the data adequately $(57,59,60,78,80)$. While the fastest absorption processes may physiologically correspond to (dissolution and) absorption in the alveolar space due to the previously mentioned physiological characteristics, the slower absorption processes may either represent GI absorption of swallowed drug (deposited in the mouth-throat region/transported to the mouth-throat region after mucociliary clearance) or pulmonary (dissolution and) absorption of drug deposited in the more central airways. However, to differentiate between slower pulmonary absorption processes and absorption processes in the GI tract, either a charcoal trial needs to be performed (59) or the oral bioavailability needs to be negligible (57).

Another model structure was presented for inhaled prochlorperazine (model subtype II-a, see Fig. 5) (78). From a physiological point of view, the shared absorption (transit) compartments for both IV and inhalational drug administration might be difficult to interpret. If the absorption compartments represent pulmonary distribution processes delaying pulmonary absorption (see discussion "Pulmonary Tissue Affinity of Drugs" above), these compartments might coincide with systemic disposition compartments. However, a model structure without redistribution from the systemic central compartment to the absorption (transit) compartments was identified (model subtype II-a).

\section{General Aspects of Empirical PK Modeling}

One important aspect of empirical modeling might be the opportunity to investigate hypotheses to explain PK characteristics after drug inhalation. Unfortunately, rarely presented in literature, the values of the parameter estimates are not discussed: for instance, one advanced approach would be correlating the absorption characteristics (e.g., fast absorbed fraction) to pulmonary deposition patterns. This approach may increase the ability to demonstrate a fast absorption process in the alveolar space or may indicate a quantitative link between the pulmonary particle deposition and the different absorbed fractions. By establishing this quantitative link, using prior mechanistic information (e.g., particle deposition patterns simulated with CFD, or particle size distribution determined with in vitro assays that demonstrated IVIVC) to develop more mechanistic empirical PK models may become feasible. These could in turn be utilized to predict the systemic PK and the absorption processes of various what-if scenarios. Given the data gaps for a comprehensive PBPK model, these semi-mechanistic models might be a very suitable alternative to PBPK approaches for answering important drug development questions, e.g., in optimizing drug formulations, devices used for drug administration, or even predict the PK of different patient populations, e.g., the prediction of the impact of a higher central 
deposited fraction on absorption processes after inhalation (slowly absorbed) in patients compared with healthy volunteers (32) or a higher peripheral fraction (fast absorbed) if a drug formulation with a higher fine particle fraction (particles $<5 \mu \mathrm{m}$ aerodynamic diameter) is inhaled.

Another challenge that might be addressed by empirical modeling is to establish a quantitative link between the (systemic) PK and the efficacy of an inhaled drug. Even though on a theoretical level, the relationship between PK parameters and efficacy has been evaluated (7), to this date not a single empirical PK/PD model has been published capable to demonstrate a relation between efficacy of a locally acting inhaled drug to systemic PK, whereas several approaches accounted for the systemic safety of inhaled drugs (see Table I). A likely explanation is that the lung as the target organ is localized before absorption to plasma, where drug concentrations are typically measured. Moreover, drug absorbed from either the lungs or the GI tract differently contributes to pulmonary efficacy, even though both absorption processes might similarly contribute to systemic exposure. Another challenge might be to use empirical models of the systemic PK to accurately characterize the pulmonary absorption processes and gain information on the pulmonary PK itself. Pulmonary PK may than contain more valuable information, which might be used for efficacy assessment.

Empirical models developed with both IV and inhalation data might also enable to differentiate between covariate effects specific for aspects of drug inhalation and systemic disposition. Some possibly relevant covariates for the inhalation parameters might be smoking, an airway disease, age, etc. By including covariates, it might be possible to further increase the understanding about the PK of inhaled drugs.

\section{Summary}

In summary, the following fundamental recommendations should be considered for empirical modeling the PK of inhaled drugs: (i) IV data is crucial to determine the total and pulmonary bioavailability and to determine if flip-flop kinetics is present, (ii) a charcoal trial or data after oral administration is necessary to differentiate between pulmonary and GI tract absorption, (iii) the impact of accounting for a high fraction of data below the LLOQ should be considered as a result of low doses administered by inhalation, (iv) the sampling scheme should be adapted to the specific characteristics of inhaled drugs (start of PK sampling directly after drug inhalation as no lag time for pulmonary absorption is expected), (v) different absorption models with, e.g., different numbers of parallel absorption processes should be investigated to increase the understanding about the pulmonary processes, and (vi) the PK model should be used to develop and test hypotheses about the physiological/mechanistic background of both systemic and pulmonary PK of inhaled drugs, i.e., to test if the pulmonary particle deposition patterns are in agreement with the model.

\section{CONCLUSIONS}

Pulmonary deposition, dissolution, and absorption are highly complex processes influencing the pulmonary and systemic PK after oral drug inhalation. These highly complex processes and still missing/incomplete or imprecise mechanistic information may represent the major challenges for building appropriate PBPK models. Both factors also increase the difficulty in interpreting the outcomes of empirical PK modeling. In addition, the challenge in linking systemic PK with pulmonary efficacy may be another factor contributing to the limited number of existing PK models for orally inhaled drugs.

Additional investigations comprising in vitro experiments, clinical studies, and more sophisticated mathematical approaches are necessary for elucidating the highly complex pulmonary deposition, dissolution, and absorption processes. With this additional knowledge, the PBPK approach might gain additional attractiveness. (Semi-)mechanistic modeling offers an alternative to generate and investigate hypotheses and to understand the pulmonary and systemic PK after drug inhalation.

\section{ACKNOWLEDGMENT}

This work was funded by Boehringer Ingelheim Pharma $\mathrm{GmbH} \& \mathrm{Co} . \mathrm{KG}$.

\section{REFERENCES}

1. Vlasakakis G, Comets E, Keunecke A, Gueorguieva I, Magni P, Terranova $\mathrm{N}$, et al. White paper: landscape on technical and conceptual requirements and competence framework in drug/ disease modeling and simulation. CPT: Pharmacometrics Syst Pharmacol. 2013;2:e40.

2. Lee JY, Garnett CE, Gobburu JV, Bhattaram VA, Brar S, Earp JC, et al. Impact of pharmacometric analyses on new drug approval and labelling decisions: a review of 198 submissions between 2000 and 2008. Clin Pharmacokinet. 2011;50(10):627-35.

3. Manolis E, Rohou S, Hemmings R, Salmonson T, Karlsson M, Milligan PA. The role of modeling and simulation in development and registration of medicinal products: output from the EFPIA/EMA modeling and simulation workshop. CPT: Pharmacometrics Syst Pharmacol. 2013;2:e31.

4. Kandala B, Hochhaus G. Pharmacometrics in pulmonary diseases. In: Schmidt S, Derendorf H, editors. Applied Pharmacometrics. 1st ed. Springer; 2014. p. 349-82.

5. Global Initiative for Chronic Obstructive Lung Disease (GOLD). Global strategy for the diagnosis, management, and prevention of chronic obstructive pulmonary disease. 2014.

6. Global Initiative for Asthma (GINA). Global strategy for asthma management and prevention. 2014.

7. Rohatagi S, Appajosyula S, Derendorf H, Szefler S, Nave B, Zech K, et al. Risk-benefit value of inhaled glucocorticoids: a pharmacokinetic/pharmacodynamic perspective. J Clin Pharmacol. 2004;44(1):37-47.

8. Jennings BH, Andersson K-E, Johansson SA. Assessment of systemic effects of inhaled glucocorticosteroids: comparison of the effects of inhaled budesonide and oral prednisolone on adrenal function and markers of bone turnover. Eur J Clin Pharmacol. 1991;40:77-82.

9. Lipworth BJ. Systemic adverse effects of inhaled corticosteroid therapy: a systematic review and meta-analysis. Arch Intern Med. 1999;159(9):941-55.

10. Hochhaus G, Möllmann H, Derendorf H, Gonzales-Rothi RJ. Pharmacokinetic/pharmacodynamic aspects of aersosol therapy using glucocorticoids as a model. J Clin Pharmacol. 1997;37(10):881-92.

11. Hollander PA, Blonde L, Rowe R, Mehta AE, Milburn JL, Hershon KS, et al. Efficacy and safety of inhaled insulin (exubera) compared with subcutaneous insulin therapy in patients with type 2 diabetes: results of a 6-month, randomized, comparative trial. Diabetes Care. 2004;27(10):2356-62. 
12. Labiris NR, Dolovich MB. Pulmonary drug delivery. Part I: physiological factors affecting therapeutic effectiveness of aerosolized medications. Br J Clin Pharmacol. 2003;56(6):588-99.

13. Olsson B, Bondesson E, Borgström L, Edsbacker S, Eirefelt S, Ekelund K, et al. Pulmonary drug metabolism, clearance, and absorption. In: Smyth HDC, Hickey AJ, editors. Controlled Pulmonary Drug Delivery. 1st ed. Springer; 2011. p. 21-50.

14. Ruge CA, Kirch J, Lehr CM. Pulmonary drug delivery: from generating aerosols to overcoming biological barriers-therapeutic possibilities and technological challenges. Lancet Respir Med. 2013;1(5):402-13.

15. Patton JS, Byron PR. Inhaling medicines: delivering drugs to the body through the lungs. Nat Rev Drug Discov. 2007;6(1):67-74.

16. Upton RN, Doolette DJ. Kinetic aspects of drug disposition in the lungs. Clin Exp Pharmacol Physiol. 1999;26(5-6):381-91.

17. International Commission on Radiological Protection. ICRP Publication 66 human respiratory tract model for radiological protection. New York: Elsevier; 1994.

18. Brown Jr RA, Schanker LS. Absorption of aerosolized drugs from the rat lung. Drug Metab Dispos. 1983;11(4):355-60.

19. Schanker LS, Mitchell EW, Brown Jr RA. Species comparison of drug absorption from the lung after aerosol inhalation or intratracheal injection. Drug Metab Dispos. 1986;14(1):79-88.

20. French MC, Wishart GN. Isolated perfused rabbit lung as a model to study the absorption of organic aerosols. J Pharmacol Methods. 1985;13:241-8.

21. Folkesson HG, Matthay MA, Westrom BR, Kim KJ, Karlsson BW, Hastings RH. Alveolar epithelial clearance of protein. J Appl Physiol. 1996;80(5):1431-45.

22. Kuempel ED, O'Flaherty EJ, Stayner LT, Smith RJ, Green FH, Vallyathan V. A biomathematical model of particle clearance and retention in the lungs of coal miners. Regul Toxicol Pharmacol : RTP. 2001;34(1):69-87.

23. Gregoratto D, Bailey MR, Marsh JW. Modelling particle retention in the alveolar-interstitial region of the human lungs. J Radiol Prot. 2010;30(3):491-512.

24. Edsbäcker S, Wollmer P, Selroos O, Borgstrom L, Olsson B, Ingelf J. Do airway clearance mechanisms influence the local and systemic effects of inhaled corticosteroids? Pulm Pharmacol Ther. 2008;21(2):247-58.

25. Isaar M, Cary M, Khan P, Hochhaus G. Pharmacokinetics and pharmacodynamics of drug delievered to the lungs. In: Hickey AJ, editor. Pharmaceutical Inhalation Aerosol Technology. 2nd ed. CRC Press; 2003. p. 215-52.

26. Carvalho TC, Peters JI, Williams 3rd RO. Influence of particle size on regional lung deposition-what evidence is there? Int $\mathrm{J}$ Pharm. 2011;406(1-2):1-10.

27. Isaacs KK, Rosati JA, Martonen TB. Mechanisms of particle deposition. In: Ruzer LS, Harley NH, editors. Aerosols Handbook. 2nd ed. New York: CRC Press; 2012. p. 47-74.

28. Labiris NR, Dolovich MB. Pulmonary drug delivery. Part II: the role of inhaland delivery devices and drug formulations in therapeutic effectiveness of aerosolized medications. Br J Clin Pharmacol. 2003;56:600-12.

29. Perrie Y, Badhan RK, Kirby DJ, Lowry D, Mohammed AR, Ouyang $\mathrm{D}$. The impact of ageing on the barriers to drug delivery. J Control Release : Off J Control Release Soc. 2012;161(2):389_ 98.

30. Withers RT, Bourdon PC, Crockett A. Lung volume standards for healthy male lifetime nonsmokers. Chest. 1988;93(1):91-7.

31. Pichelin M, Caillibotte G, Katz I, Martonen T. Categorization of lung morphology based on FRC and height: computer simulations of aerosol deposition. Aerosol Sci Technol. 2012;46:70-81.

32. Darquenne C. Aerosol deposition in health and disease. J Aerosol Med Pulm Drug Deliv. 2012;25(3):140-7.

33. Brutsche MH, Brutsche IC, Munavvar M, Langley SJ, Masterson $\mathrm{CM}$, Daley-Yates $\mathrm{P}$, et al. Comparison of pharmacokinetic and systemic effects of inhaled fluticasone proprionate in patients with asthma and healthy volunteers: a randomised crossover study. Lancet. 2000;356:556-61.

34. Singh SD, Whale C, Houghton N, Daley-Yates P, Woodcock AA. Pharmacokinetic and systemic effects of inhaled fluticasone propionate in chronic obstructive pulmonary disease. Br J Clin Pharmacol. 2003;55:375-81.
35. Weiner P, Berar-Yanay N, Davidovich A, Magadle R. Nocturnal cortisol secretion in asthmatic parients after inhalation of fluticasone propionate. Chest. 1999;116(4):931-4.

36. Mortimer KJ, Tattersfield AE, Tang Y, Wu K, Lewis S, Hochhaus $\mathrm{G}$, et al. Plasma concentrations of fluticasone propionate and budesonide following inhalation: effect of induced bronchoconstriction. $\mathrm{Br} \mathrm{J}$ Clin Pharmacol. 2007;64(4):439-44.

37. Imai Y, Miki T, Ishikawa T, Aoki T, Yamaguchi T. Deposition of micrometer particles in pulmonary airways during inhalation and breath holding. J Biomech. 2012;45(10):1809-15.

38. Zhao P, Zhang L, Grillo JA, Liu Q, Bullock JM, Moon YJ, et al. Applications of physiologically based pharmacokinetic (PBPK) modeling and simulation during regulatory review. Clin Pharmacol Ther. 2011;89(2):259-67.

39. Jones HM, Gardner IB, Watson KJ. Modelling and PBPK simulation in drug discovery. AAPS J. 2009;11(1):155-66.

40. Bonate PL. Pharmacokinetic-pharmacodynamic modeling and simulation. 2nd ed. Springer Science and Business Media; 2011.

41. Landersdorfer CB, Jusko WJ. Pharmacokinetic/ pharmacodynamic modeling of glucose clamp effects of inhaled and subcutanous insulin in healthy volunteers and diabetic patients. Drug Metab Pharmacokinet. 2010;25(5):418-29.

42. Sakagami M. Insulin disposition in the lung following oral inhalation in humans: a meta-analysis of its pharmacokinetics. Clin Pharmacokinet. 2004;43(8):539-52.

43. Gloede E, Cichocki JA, Baldino JB, Morris JB. A validated hybrid computational fluid dynamics-physiologically based pharmacokinetic model for respiratory tract vapor absorption in the human and rat and its application to inhalation dosimetry of diacetyl. Toxicol Sci : Off J Soc Toxicol. 2011;123(1):231-46.

44. Morris JB. Biologically-based modeling insights in inhaled vapor absorption and dosimetry. Pharmacol Ther. 2012;136(3):401-13.

45. Gaz C, Cremona G, Panunzi S, Patterson B, De Gaetano A. A geometrical approach to the PKPD modelling of inhaled bronchodilators. J Pharmacokinet Pharmacodyn. 2012;39(5):415-28.

46. Applied Research Associates. Multiple-path dosimetry model 2009 [cited 201414 Oct]. Available from: http://www.ara.com/ products/mppd.

47. Meltzer EO, Kuna P, Nolte H, Nayak AS, Laforce C. Mometasone furoate/formoterol reduces asthma deteriorations and improves lung function. Eur Respir J. 2012;39(2):279-89.

48. Chaudhuri SR, Lukacova V. Simulating delivery of pulmonary (and intranasal) aerosolised drugs. Orally Inhaled Nasal Drug Prod. 2010:26-30.

49. Collingwood SP, Coe D, Pryde D, Lock R. Respiratory drug discovery, current developments and future challenges: highlights from the Society of Medicines Research Symposium, held on June 14 th, 2012-Horsham, UK. Drugs Future. 2012;37(8):61925 .

50. Agoram B, Woltosz WS, Bolger MB. Predicting the impact of physiological and biochemical processes on oral drug bioavailability. Adv Drug Deliv Rev. 2001;50 Suppl 1:S41-67.

51. Noyes AA, Whitney WR. The rate of solution of solid substances in their own solutions. J Am Chem Soc. 1897;19:930-4.

52. Chaudhuri SR, Lukacova V, Woltosz WS. Application of a respiratory PBPK model for predicting deposition and disposition following inhaled administration of morphine. AAPS Annual Meeting and Exposition/FIP Pharmaceutical Sciences 2010 World Congress; 14-18 Nov 2010; New Orleans, LA2010.

53. Chaudhuri SR, Lukacova V, Woltosz WS, editors. Simulating the disposition of triamcinolone acetonide following oral and pulmonary administration. Respiratory Drug Delivery Europe 2011; 2011 3-6 May 2011; Berlin, Germany: Davis Healthcare International Publishing, LLC.

54. Miller NA, Chaudhuri SR, Lukacova V, Damian-Iordache V, Bayliss MK, Woltosz WS, editors. Development of a physiologically based pharmacokinetic (PBPK) model for predicting deposition and disposition following inhaled and intranasal administration. Respiratory Drug Delivery 2010; 2010 25-29 Apr 2010; Orlando, Florida: Davis Healthcare International Publishing, LLC. 
55. Lukacova V, Chaudhuri SR, Miller NA, Damian-Iordache V, Bolger MB, Woltosz WS, editors. Simulation of tobramycin pharmacokinetics after pulmonary administration. 37th Annual Meeting \& Exposition of the Controlled Release Society 2010; 2010 10-14 Jul 2010; Portland, Oregon.

56. Simcyp Limited. Lung absorption [cited 20146 Jun]. Available from: http://www.simcyp.com/ResearchDevelopment/ SimcypScience/Absorption/Pulmonary+absorption/.

57. Borghardt JM, Weber B, Staab A, Kunz C, Schiewe J, Kloft C. Expanding the mechanistic knowledge about pulmonary absorption processes using a population pharmacokinetic model for inhaled olodaterol. Respiratory Drug Delivery; 2014; Fajardo, Puerto Rico: Davis Healthcare International Publishing, LLC.

58. Parra-Guillen Z, Weber B, Sharma A, Freijer J, Retlich S, Borghardt JM, et al. Population pharmacokinetic analysis of tiotropium in healthy volunteers after intravenous administration and inhalation. J Pharmacokinet Pharmacodyn. 2014;41(1):S54.

59. Bartels C, Looby M, Sechaud R, Kaiser G. Determination of the pharmacokinetics of glycopyrronium in the lung using a population pharmacokinetic modelling approach. Br J Clin Pharmacol. 2013;76(6):868-79.

60. Dershwitz M, Walsh JL, Morishige RJ, Connors PM, Rubsamen RM, Shafer SL, et al. Pharmacokinetics and pharmacodynamics of inhaled versus intravenous morphine in healthy volunteers. Anesthesiology. 2000;93(3):619-28.

61. Rohatagi S, Krishnaswami S, Pfister M, Sahasranaman S. Modelbased covariate pharmacokinetic analysis and lack of cortisol suppression by the new inhaled corticosteroid ciclesonide using a novel cortisol release model. Am J Ther. 2005;12:385-97.

62. Rohatagi S, Arya V, Zech K, Nave R, Hochhaus G, Jensen BK, et al. Population pharmacokinetics and pharmacodynamics of ciclesonide. J Clin Pharmacol. 2003;43(4):365-78.

63. Xu J, Nave R, Lahu G, Derom E, Derendorf H. Population pharmacokinetics and pharmacodynamics of inhaled ciclesonide and fluticasone propionate in patients with persistent asthma. J Clin Pharmacol. 2010;50(10):1118-27.

64. Minto C, Li B, Tattam B, Brown K, Seale JP, Donnelly R. Pharmacokinetics of epimeric budesonide and fluticasone propionate after repeat dose inhalation-intersubject variability in systemic absorption from the lung. $\mathrm{Br} \mathrm{J}$ Clin Pharmacol. 2000;50(2):116-24.

65. Wu K, Goyal N, Stark JG, Hochhaus G. Evaluation of the administration time effect on the cumulative cortisol suppression and cumulative lymphocytes suppression for once-daily inhaled corticosteroids: a population modeling/simulation approach. J Clin Pharmacol. 2008;48(9):1069-80.

66. Krishnaswami S, Hochhaus G, Möllmann H, Barth J, Derendorf $\mathrm{H}$. Interpretation of absorption rate data for inhaled fluticasone propionate obtained in compartmental pharmacokinetic modeling. Int J Clin Pharmacol Ther. 2005;43(3):117-22.

67. Blake K, Mehta R, Spencer T, Kunka RL, Hendeles L. Bioavailability of inhaled fluticasone propionate via chambers/ masks in young children. Eur Respir J. 2012;39(1):97-103.

68. Anderson PJ, Zhou X, Breen P, Gann L, GLogsdon TW, Compadre CM, et al. Pharmacokinetics of (R, S)-albuterol after aerosol inhalation in healthy adult volunteers. J Pharm Sci. 1998;87(7):841-4.

69. Maier G, Rubino C, Hsu R, Grasela T, Baumgartner RA. Population pharmacokinetics of (R)-albuterol and (S)-albuterol in pediatric patients aged 4-11 years with asthma. Pulm Pharmacol Ther. 2007;20(5):534-42.

70. Derks MG, van den Berg BT, van der Zee JS, Braat MC, van Boxtel CJ. Biphasic effect-time courses in man after formoterol inhalation: eosinopenic and hypokalemic effects and inhibition of allergic skin reactions. J Pharmacol Exp Ther. 1997;283(2):82432.

71. Matsushima S, Matthews I, Woessner R, Pinault G, Hara H, Wilkins $\mathrm{J}$, et al. Systemic pharmacokinetics of indacaterol, an inhaled once-daily long-acting beta2-agonist, in different ethnic populations. Int J Clin Pharmacol Ther. 2012;50(8):545-56.

72. Diderichsen PM, Cox E, Martin SW, Cleton A, Ribbing J. Characterizing systemic exposure of inhaled drugs: application to the long-acting beta2-agonist PF-00610355. Clin Pharmacokinet. 2013;52(6):443-52.
73. Peng AW, Hussey EK, Moore KH. A population pharmacokinetic analysis of zanamivir in subjects with experimental and naturally occurring influenza: effects of formulation and route of administration. J Clin Pharmacol. 2000;40(3):242-9.

74. Yoshihara K, Ishizuka H, Kubo Y. Population pharmacokinetics of laninamivir and its prodrug laninamivir octanoate in healthy subjects and in adult and pediatric patients with influenza virus infection. Drug Metab Pharmacokinet. 2013;28(5):416-26.

75. Marino MT, Costello D, Baughman R, Boss A, Cassidy J, Damico C, et al. Pharmacokinetics and pharmacodynamics of inhaled GLP-1 (MKC253): proof-of-concept studies in healthy normal volunteers and in patients with type 2 diabetes. Clin Pharmacol Ther. 2010;88(2):243-50.

76. Ting L, Aksenov S, Bhansali SG, Ramakrishna R, Tang P, Geller DE. Population pharmacokinetics of inhaled tobramycin powder in cystic fibrosis patients. CPT: Pharmacometrics Syst Pharmacol. 2014:3:e99.

77. Shah B, Jensen BK, Zhang J, Hunt T, Rohatagi S. Effect of food on pharmacokinetics of an inhaled drug: a case study with a VLA-4 antagonist, HMR1031. J Clin Pharmacol. 2003;43(12):1341-9.

78. Avram MJ, Spyker DA, Henthorn TK, Cassella JV. The pharmacokinetics and bioavailability of prochlorperazine delivered as a thermally generated aerosol in a single breath to volunteers. Clin Pharmacol Ther. 2009;85(1):71-7.

79. Potocka E, Baughman RA, Derendorf H. Population pharmacokinetic model of human insulin following different routes of administration. J Clin Pharmacol. 2011;51(7):1015-24.

80. Stass H, Nagelschmitz J, Willmann S, Delesen H, Gupta A, Baumann S. Inhalation of a dry powder ciprofloxacin formulation in healthy subjects: a phase I study. Clin Drug Investig. 2013;33(6):419-27.

81. Weber B, Hochhaus G. A pharmacokinetic simulation tool for inhaled corticosteroids. AAPS J. 2013;15(1):159-71.

82. Cheng YS. Modeling aerosol drug delivery. In: Gradon L, editor. Optimization of aerosol drug delivery. 1st ed. Netherlands: Springer; 2003. p. 165-88.

83. Longest PW, Hindle M, Choudhuri SD, Xi J. Comparison of ambient and spray aerosol in a standard induction port and more realistic mouth-throat geometry. Aerosol Sci. 2008;39:572-91.

84. DeHaan WH, Finlay WH. Predicting extrathoracic deposition from dry powder inhalers. Aerosol Sci. 2004;35:309-31.

85. Wong W, Fletcher DF, Traini D, Chan HK, Crapper J, Young PM. Particle aerosolisation and break-up in dry powder inhalers 1: evaluation and modelling of venturi effects for agglomerated systems. Pharm Res. 2010;27(7):1367-76.

86. Longest PW, Holbrook LT. In silico models of aerosol delivery to the respiratory tract-development and applications. Adv Drug Deliv Rev. 2012;64(4):296-311.

87. Cuddihy RG, Fisher GL, Kanapilly GM, Moss OR, Phalen RF, Schlesinger RB, et al. Report No. 125-deposition, retention and dosimetry of inhaled radioactive substances. NCRP; 1997.

88. MacIntyre AC, Cutler DJ. The potential role of lysosomes in tissue distribution of weak bases. Biopharm Drug Dispos. 1988;9(6):513-26.

89. Vauquelin G, Charlton SJ. Long-lasting target binding and rebinding as mechanisms to prolong in vivo drug action. $\mathrm{Br} \mathrm{J}$ Pharmacol. 2010;161(3):488-508.

90. Anderson GP, Linden A, Rabe KF. Why are long-acting betaadrenoceptor agonists long-acting? Eur Respir J. 1994;7(3):56978.

91. Olsson B, Borgstrom L, Lundback H, Svensson M. Validation of a general in vitro approach for prediction of total lung deposition in healthy adults for pharmaceutical inhalation products. J Aerosol Med Pulm Drug Deliv. 2013;26(6):355-69.

92. Neale MG, Brown K, Foulds RA, Lal S, Morris DA, Thomas D. The pharmacokinetics of nedocromil sodium, a new drug for the treatment of reversible obstructive airways disease, in human volunteers and patients with reversible obstructive airways disease. Br J Clin Pharmacol. 1987;24(4):493-501.

93. Beal SL. Ways to fit a PK model with some data below the quantification limit. J Pharmacokinet Pharmacodyn. 2001;28(5):481-504.

94. Hochhaus G, Schmidt E-W, Rominger KL, Möllmann H. Pharmacokinetik/dynamic correlation of pulmonary and cardiac 
effects of fenoterol in asthmatic patients after different routes of administration. Pharm Res. 1992;9(3):291-7.

95. Patton JS, Brain JD, Davies LA, Fiegel J, Gumbleton M, Kim $\mathrm{KJ}$, et al. The particle has landed-characterizing the fate of inhaled pharmaceuticals. J Aerosol Med Pulm Drug Deliv. 2010;23 Suppl 2:S71-87.

96. Agu RU, Ugwoke MI. In vitro and in vivo testing methods for respiratory drug delivery. Expert Opin Drug Deliv. 2011;8(1):5769.

97. Tolman JA, Williams 3rd RO. Advances in the pulmonary delivery of poorly water-soluble drugs: influence of solubilization on pharmacokinetic properties. Drug Dev Ind Pharm. 2010;36(1):1-30.

98. Okusanya OO, Bhavnani SM, Hammel JP, Forrest A, Bulik CC, Ambrose PG, et al. Evaluation of the pharmacokinetics and pharmacodynamics of liposomal amikacin for inhalation in cystic fibrosis patients with chronic pseudomonal infections using data from two phase 2 clinical studies. Antimicrob Agents Chemother. 2014;58(9):5005-15.

99. Burmeister Getz E, Fisher DM, Fuller R. Human pharmacokinetics/ pharmacodynamics of an interleukin-4 and interleukin-13 dual antagonist in asthma. J Clin Pharmacol. 2009;49(9):1025-36. 